\title{
Herdabilidade da habilidade de permanência no rebanho em fêmeas de bovinos da raça Canchim
}

\author{
Leonardo Martin Nieto(1), Luiz Otavio Campos da Silva(2), Cintia Righetti Marcondes(3), \\ Antônio do Nascimento Rosa ${ }^{(2)}$, Elias Nunes Martins ${ }^{(4)}$ e Roberto Augusto de Almeida Torres Júnior ${ }^{(2)}$
}

\begin{abstract}
(1)Fundação de Apoio ao Desenvolvimento do Ensino, Ciência e Tecnologia do Estado de Mato Grosso do Sul, Rua Jornalista Belizario Lima 403, Apto. 102, CEP 79004-270 Campo Grande, MS. E-mail: Imnietogp@cnpgc.embrapa.br (2)Embrapa Gado de Corte, BR 262, Km 4, Caixa Postal 154, CEP 79002-970 Campo Grande, MS. E-mail: locs@cnpgc.embrapa.br, anrosa@cnpgc.embra.br, rtorres@cnpgc.embrapa.br ${ }^{(3)}$ Embrapa Amazônia Oriental, Av. Dr. Enéas Pinheiro, s/no, Marco, CEP 66095-100 Belém, PA. E-mail: cimarcon@cpatu.embrapa.br (4)Universidade Estadual de Maringá, Dep. de Zootecnia, Av. Colombo, o⒌790, Zona 7, CEP 87020-900 Maringá, PR. E-mail: enmartins@uem.br
\end{abstract}

\begin{abstract}
Resumo - O objetivo deste trabalho foi avaliar a possibilidade de a característica habilidade de permanência no rebanho de matrizes ser utilizada como critério de seleção na raça Canchim. A habilidade de permanência no rebanho foi definida como a probabilidade de uma matriz ter três partos ou mais até os 76 meses de idade, desde que ela tenha tido a oportunidade de se reproduzir pelo menos uma vez. Codificações binárias, com 0 para indicar fracasso e 1 para indicar sucesso, foram feitas para cada fêmea. Uma codificação alternativa, com quatro categorias, considerou os valores $0,1,2$ e 3 , respectivamente, para vacas com menos de três, com três, quatro ou cinco partos até os 76 meses de idade. As análises foram processadas pela aplicação de um modelo unicaracterístico de touro avô-materno. Utilizou-se a amostragem de Gibbs para estimar os componentes de variância e herdabilidades. As estimativas de herdabilidade foram de 0,07 , para a característica definida pelo modelo binário, e de 0,08 , para a característica definida pelo modelo com quatro categorias. Os resultados indicam pequenas diferenças na estimativa de herdabilidade, pelos modelos binário e com quatro categorias. Sugerem também que as estimativas de herdabilidade, para a característica habilidade de permanência, na raça Canchim, apresenta baixo potencial de resposta à seleção.
\end{abstract}

Termos para indexação: característica reprodutiva, gado de corte, modelo de limiar, parâmetros genéticos.

\section{Heritability for stayability in Canchim beef cattle breed}

\begin{abstract}
The objective of this work was to evaluate the effectiveness of using the stayability of beef cows as selection criterion for the Canchim breed. Stayability was defined as the probability of a beef cow having three or more parities up to 76 months of age, given that she have calved at least once and was older than 76 months. Binary coding, with 0 indicating failure and 1 indicating success, was defined for each cow. An alternative coding, with four categories, used the values $0,1,2$ and 3, respectively, for cows with less than three, three, four or five parities up to 76 months of age. The analises were perfomed with a single-trait sire and maternal grandsire model. Gibbs sampling was used to estimate variance components and heritabilities. The estimated heritability was 0.07 for the binary trait and 0.08 for the trait with the four categories. The results indicate small diferences in the estimated heritability for the binary and four-category models. They also suggest that the stayability in Canchim breed presents low potential for response to selection.
\end{abstract}

Index terms: reprodutive trait, beef cattle, treshold model, genetic parameters.

\section{Introdução}

Apesar de as características relacionadas à reprodução serem as que mais influenciam a eficiência econômica dos sistemas de produção (Meacham \& Notter, 1987), raramente elas foram utilizadas como critérios de seleção nos programas de melhoramento estabelecidos, especialmente a partir das décadas de 80 e 90. Entre as razões para este fato, sem dúvida, devem ser consideradas as dificuldades de sua aferição em condições de campo, e a carência de estudos que determinam as correlações genéticas entre estas variáveis e aquelas relacionadas diretamente aos objetivos do sistema de produção, tais como peso corporal e taxa de crescimento. Rebanhos férteis, sexualmente precoces e com rápido acabamento de carcaça apresentam maior oferta de animais, tanto para a venda 
de produtos comerciais para recria, engorda ou abate quanto para o trabalho de melhoramento, o que permite maior intensidade de seleção e, conseqüentemente, maiores progressos genéticos e lucratividade.

Na maioria das vezes, os parâmetros genéticos para características de desempenho reprodutivo de fêmeas, como idade ao primeiro parto, duração da gestação e dias para parir, são obtidos por meio de aplicações de modelos lineares que assumem distribuição normal dos dados (Pereira et al., 2001; Dias et al., 2004)

Algumas características reprodutivas, no entanto, são do tipo categórico de limiar ( o desenvolvimento de procedimentos analíticos para dados categóricos ser recente, características deste tipo, medidas diretamente nas fêmeas, têm sido até agora pouco exploradas em termos práticos. Esses procedimentos, embora mais complexos do que os utilizados para características representadas por variáveis contínuas, podem possibilitar a identificação de novas características. Recentemente, a habilidade de permanência no rebanho (HP) ou "stayability", definida como a probabilidade de as fêmeas se encontrarem em fase reprodutiva a uma idade específica, dado que tiveram a oportunidade de alcançar esta idade (Hudson \& Vleck, 1981), tem recebido especial atenção por parte dos pesquisadores. Segundo Silva et al. (2003), a inclusão desta característica, nos programas de melhoramento genético, permitiria a seleção de reprodutores que produziriam filhas com maior probabilidade de permanecerem produtivas no rebanho por um período mais longo de tempo.

No Brasil, há estudos sobre permanência no rebanho com a raça Nelore. Silva et al. (2003) obtiveram estimativa de 0,21 para HP, definida como a probabilidade de uma vaca parir, no rebanho, até a idade de seis anos ou mais, desde que ela tenha tido uma parição anterior. Marcondes et al. (2005) obtiveram estimativa de 0,16 para HP, com um modelo de limiar, e de 0,13 com modelo linear. Ao estudar as relações genéticas entre HP, circunferência escrotal e ganho pós-desmama, Silva et al. (2006) estimaram a herdabilidade de 0,22 para HP. Recentemente, Melis et al. (2007) estimaram herdabilidade de 0,25, 0,22 e 0,28 para HP aos cinco, seis e sete anos de idade, respectivamente.

O objetivo deste trabalho foi estimar a herdabilidade da característica habilidade de permanência no rebanho, para a raça Canchim, tendo-se empregado duas definições para esta característica.

\section{Material e Métodos}

A partir da base de dados do Programa de Melhoramento Genético Embrapa-Geneplus, para a raça Canchim, foi gerado um arquivo de dados para a característica habilidade de permanência no rebanho (HPR), com os registros de matrizes nascidas entre os anos de 1986 e 1999.

As matrizes que tiveram três partos ou mais, até os 76 meses de idade, foram codificadas com valor 1 , enquanto as demais, que tiveram menos de três partos, foram codificadas com valor zero 0 . Os grupos contemporâneos foram constituídos por animais nascidos na mesma fazenda e no mesmo ano.

Os grupos com médias zero ou um, para a característica em estudo, isto é, sem variabilidade dentro do grupo de contemporâneos, foram identificados por meio do SAS (SAS Institute, 2000) e foram eliminados do banco de dados.

Alternativamente, com o objetivo de identificar a variabilidade genética existente para a característica HPR, foi gerado um novo arquivo, no qual foram atribuídos valores $0,1,2$ e 3 para vacas com menos de três partos, e com três, quatro ou cinco partos até os 76 meses de idade, respectivamente.

As estimativas dos componentes de variância e dos parâmetros genéticos para longevidade, avaliada pela característica habilidade de permanência no rebanho, foram obtidas por meio do modelo de limiar, com emprego do programa MTGSAM-THR (Tassell et al., 1998), sob modelo unicaráter de touro-avô materno, ante a impossibilidade de se implementar o modelo animal, por problemas de convergência.

O conceito de limiar (Falconer, 1965) pressupõe que as respostas estejam relacionadas a uma variável normal subjacente, usualmente chamada "liability" ( $)$ e a um conjunto de limiares fixos, que dividem a linha real em intervalos $\mathrm{m}$, correspondentes às categorias de resposta.

Pressupõe-se que os dados são organizados numa tabela s x m, onde as linhas s representam combinações de variáveis explanatórias, e as colunas m indicam categorias de resposta (Gianola \& Foulley, 1983).

As entradas nessa tabela $n_{j k}$ são feitas pelo número de observações na $\mathrm{k}^{\text {ésima }}$ categoria de resposta $(\mathrm{k}=1,2$ ..., $\mathrm{m})$ e na jésima linha $(\mathrm{j}=1,2 \ldots, \mathrm{s})$. Os totais de linhas nj são considerados fixos.

A "liability", ou predisposição, pode ser modelada como: $\vartheta_{\mathrm{jq}}=\eta j+\varepsilon_{\mathrm{jq}}$, em que: $\eta_{\mathrm{j}}$ é um parâmetro de locação para a jésima combinação de variáveis explanatórias, $\mathrm{j}=1,2, \ldots$, $\mathrm{s} ; \mathrm{q}=1,2, \ldots \eta_{\mathrm{j}}$ e $\varepsilon_{\mathrm{jq}} \sim \mathrm{N}(0,1)$. 
Aos parâmetros de locação $\eta_{\mathrm{j}}$ é dada uma estrutura linear, como a do Modelo Touro. Assim, o modelo acima pode ser escrito: $\vartheta_{\mathrm{jq}}=\eta \mathrm{j}+\varepsilon_{\mathrm{jq}}=\mathrm{W}_{\mathrm{jq}} \theta_{\mathrm{t}}+\varepsilon_{\mathrm{jq}}$, em que: $\vartheta_{\mathrm{jq}}$ é uma variável contínua subjacente do $\mathrm{k}^{\text {ésimo }}$ animal, na jésima combinação; $W_{\mathrm{jq}}$ é um vetor de incidência para a jq ${ }^{\text {ésima }}$ observação; e $\theta_{t}$ contém os mesmos efeitos fixos e aleatórios do modelo linear.

Foram definidas cadeias de 550 mil ciclos, e foi tomada uma amostra a cada 500 ciclos, após os primeiros $50 \mathrm{mil}$ ciclos terem sido desprezados. O procedimento de retiradas das amostras a grandes intervalos assegura que a correlação entre elas seja nula ou muito pequena (Tassell \& Vleck, 1996). Foram obtidas, assim, 1.000 amostras para cada parâmetro a ser estimado, o que permitiu a elaboração dos gráficos, após análises dos dados das amostras por meio do programa Gibanal (Van Kaam, 1997) para a visualização das distribuições dos componentes em cada análise, bem como o estabelecimento de intervalos de credibilidade a $95 \%$ das distribuições posteriores.

A herdabilidade estimada com o modelo touro-avô materno foi calculada como a fração das diferenças fenotípicas que é de origem genética; a variância genética aditiva foi considerada como $4 \sigma_{\mathrm{s}}^{2}$ (variância de touro), a variância ambiental como sendo igual à variância residual $\left(\sigma_{\mathrm{e}}^{2}\right)$, no caso de modelo de limiar, fixa com valor 1, e a variância fenotípica como a soma de $\left(\sigma_{\mathrm{s}}^{2}+1 / 4 \sigma_{\mathrm{s}}^{2}\right)$ à variância residual.

\section{Resultados e Discussão}

As estatísticas descritivas para as características definidas pelo modo padrão foram: de um total de 24.927 observações, obtiveram-se 12.329 sucessos $(49,46 \%)$. No modo alternativo, observaram-se os seguintes resultados: para o valor atribuído à HPR de 0 , obtiveram-se 12.598 observações - freqüência $(\mathrm{F})=50,54 \%$; para o valor $1,5.026-\mathrm{F}=20,16 \%$; para o valor $2,4.249-\mathrm{F}=17,05 \%$; para o valor 3, $3.054-$ $\mathrm{F}=12,25 \%$.
Na Tabela 1, podem ser observadas as estatísticas descritivas, para as estimativas dos componentes genéticos e da herdabilidade obtidas na análise da característica HPR quando codificada na forma-padrão e alternativa.

Para forma-padrão, os valores da média, moda e da mediana, para a herdabilidade de HPR, foram muito próximos, o que indica que as medidas de tendência central foram relativamente simétricas, e isto pode ser confirmado pela distribuição marginal apresentada na Figura 1.

O valor de herdabilidade média obtida para HPR, aos 76 meses de idade, pode ser considerado baixo. Westhuizen et al. (2001) estudaram a HPR, por meio de modelo touro, a qual foi definida como a probabilidade de um animal sobreviver a uma idade específica $(36,48$, 60,72 e 84 meses), dada a oportunidade de atingir aquela idade, e obtiveram estimativas de herdabilidade de 0,06 , $0,10,0,06,0,03$ e 0,11 , respectivamente. Os autores concluíram que o ganho genético para HPR seria limitado e, também, que os touros tiveram pequena influência sobre a característica em estudo. Esses mesmos autores destacam que esses resultados poderiam ser diferentes, caso os dados fossem avaliados sob modelo animal.

Estudos realizados com outras raças reportaram herdabilidades moderadas para HPR. Em relação à raça Angus, Doyle et al. (2000) obtiveram estimativa de herdabilidade de 0,15 , para vacas que tiveram cinco crias, dado que tiveram duas crias até os dois anos de idade. Martinez et al. (2004) estudaram a HPR em vacas Hereford, por meio de modelo touro, e reportaram herdabilidade de 0,09 a 0,17.

Em relação à raça Nelore, Silva et al. (2003) e Marcondes et al. (2005) relataram valores semelhantes $(0,15)$ para habilidade de permanência até cinco anos, dado que a vaca tinha produzido pelo menos uma cria antes desta idade, e para habilidade de permanência até seis anos, dado que a vaca tinha produzido três crias. Silva et al. (2006) estimaram herdabilidade de 0,22 , para a habilidade de permanência no rebanho.

Tabela 1. Estatísticas descritivas da variância de touro $\left(\sigma_{\mathrm{s}}^{2}\right)$ e da herdabilidade $\left(\mathrm{h}^{2}\right)$, para a característica habilidade de permanência no rebanho da raça Canchim, quando codificada na forma-padrão (PD) e na forma alternativa (AL).

\begin{tabular}{lccccccccc}
\hline Estatística & Forma & Média & Moda & Mediana & Mínimo & Máximo & 0,020 & 0,038 & $0,008-0,027$ \\
\hline$\sigma_{s}^{2}$ & PD & 0,017 & 0,017 & 0,017 & 0,008 & 0,036 \\
\hline $\mathrm{h}^{2}$ & AL & 0,021 & 0,021 & 0,021 & $0,013-0,030$ \\
& PD & 0,066 & 0,066 & 0,065 & 0,008 & 0,146 \\
& AL & 0,083 & 0,082 & 0,082 & 0,033 & 0,139 & $0,052-0,116$ \\
\hline
\end{tabular}

(1)IC: intervalo de credibilidade a 95\%. 
Melis et al. (2007) estudaram as relações genéticas da característica habilidade de permanência no rebanho, quando avaliada em diferentes idades, e obtiveram herdabilidades entre 0,22 e 0,28 .

Segundo Silva et al. (2003), a utilização da HPR, como critério de seleção dos animais que entraram em reprodução, conduziria ao aumento no tempo médio de permanência no rebanho, e diminuiria a necessidade de novilhas de reposição. Além disso, este procedimento conduziria a um maior intervalo de gerações, que seria agravado ainda mais pelo fato de que touros jovens, por apresentarem baixa acurácia para HPR até as filhas atingirem uma determinada idade, seriam pouco utilizados na reprodução. Este problema poderia ser amenizado pelo uso de análise de sobrevivência, em lugar dos modelos de limiar. A análise de sobrevivência considera a permanência de uma forma contínua e retém mais informação, além de considerar dados censurados de animais que ainda estão vivos e não atingiram o limite de idade adotado. Isso, no entanto, não muda o fato de esta ser uma característica reprodutiva que começa a ser expressa por volta dos 24 meses de idade das fêmeas filhas do touro, além do que a permanência só pode ser avaliada razoavelmente, em suas filhas, quando elas
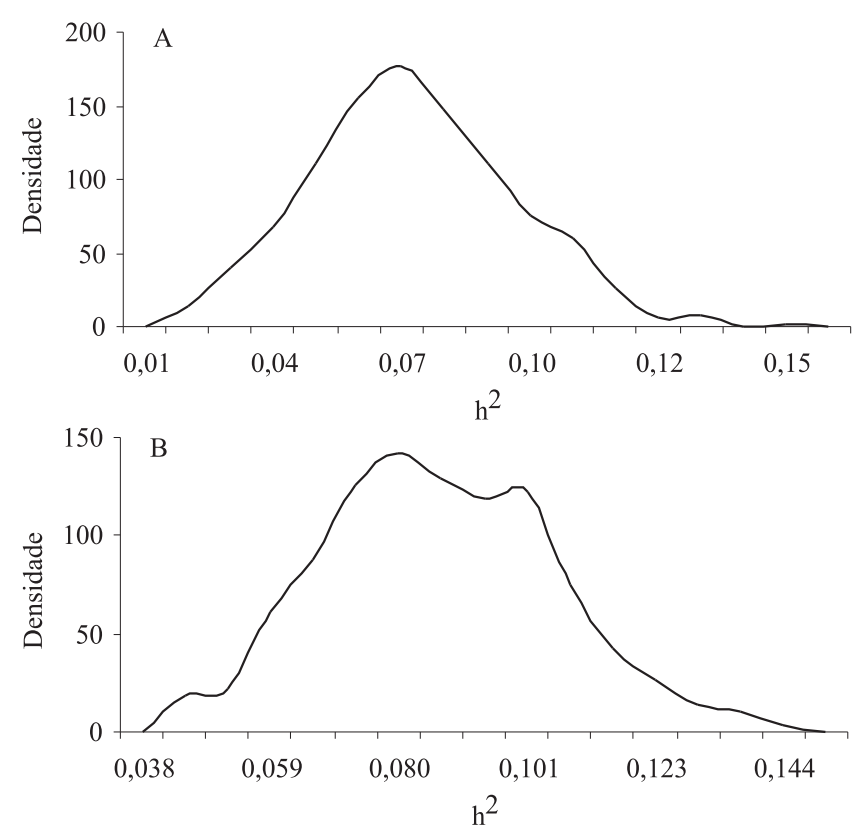

Figura 1. Distribuição posterior da estimativa de herdabilidade, para a característica habilidade de permanência no rebanho da raça Canchim, quando codificada na forma binária (A) e na forma alternativa (B). tiverem passado por uma oportunidade substancial de descarte, o que levaria um ou dois anos.

Segundo Silva et al. (2003), esta situação também poderia ser melhorada, a se analisar a HPR em uma idade menor, o que possibilitaria maior acurácia das predições dos méritos genéticos, como consequiência de um número maior de observações no arquivo de dados e diminuição do intervalo entre gerações. Este fato foi corroborado por Melis et al. (2007) ao estudar a habilidade de permanência no rebanho de vacas Nelore, aos cinco (Stay5), seis (Stay6) e sete anos de idade (Stay7). Os autores observaram relação linear entre as características, o que indicaria que a seleção, para melhorar características associadas às fêmeas, seria mais eficiente se baseada na característica avaliada aos cinco anos de idade (Stay5).

É importante ressaltar que, no presente estudo, a HPR foi definida como probabilidade das matrizes terem três partos ou mais até os 76 meses de idade, uma vez que, com três partos, a vaca já teria pagado os seus custos de cria/recria (Formigoni, 2002). Ademais, a idade de 76 meses foi estabelecida de modo que, em razão da diversidade de manejo nos diferentes rebanhos que compunham a base de dados, fosse assegurada a chance de a vaca conseguir três partos pelo menos.

Embora a HPR codificada na forma alternativa tenha permitido detectar uma maior variabilidade genética entre os indivíduos, como consequiência do aumento na variância de touro, a estimativa da herdabilidade (Tabela 1) indica que a resposta à seleção e os ganhos genéticos esperados não serão muito elevados.

A distribuição gráfica da distribuição posterior da herdabilidade para HPR (Figura 1 B), a diferencia da observada na característica-padrão (Figura $1 \mathrm{~A}$ ) e apresenta curva com distribuição bimodal $(0,08$ e 0,10$)$.

Marcondes et al. (2005) estudaram uma definição alternativa de HPR, em comparação à definição usual, por meio de um modelo linear, e obtiveram estimativas de 0,07 e 0,08, respectivamente. Estes mesmos autores concluíram que a característica habilidade de permanência no rebanho, definida pela forma alternativa, apresenta maior capacidade de detecção de variabilidade genética que a característica-padrão.

\section{Conclusões}

1. A definição alternativa para a característica habilidade de permanência mostrou-se mais eficiente do 
que a definição usual, para a detecção de variabilidade genética, embora esta variabilidade tenha sido considerada pequena.

2. As estimativas de herdabilidade indicam que a característica habilidade de permanência, na raça Canchim, apresenta baixo potencial de resposta à seleção.

\section{Referências}

DIAS, L.T.; EL FARO, L.; ALBURQUERQUE, L.G. Estimativas de herdabilidade para idade ao primeiro parto de novilhas da raça Nelore. Revista Brasileira de Zootecnia, v.33, p.97-102, 2004.

DOYLE, S.P.; GOLDEN, B.L.; GREEN, R.D.; BRINKS, J.S. Additive genetic parameter estimates for heifer pregnancy and subsequent reproduction in Angus females. Journal of Animal Science, v.78, p.2091-2098, 2000.

FALCONER, D.S. The inheritance of liability to certain diseases estimated from the incidence from relatives. Annals of Human Genetics, v.29, p.51-76, 1965.

FORMIGNONI, I.B. Estimação de valores econômicos para características componentes de índices de seleção em bovinos de corte. 2002. 77p. Dissertação (Mestrado) - Universidade de São Paulo, Pirassununga.

GIANOLA, D.; FOULLEY, J.L. Sire evaluation for ordered categorical data with a threshold model. Genetic Selection Evolution, v.15, p.201-224, 1983.

HUDSON, G.F.S.; VLECK, L.D. van. Relations between production and stayability in Holstein cattle. Journal of Dairy Science, v.64, p.2246-2250, 1981

KAAM, J.B.C.H.M. van. GIBANAL: analyzing program for Markov Chain Monte Carlo sequences. Wageningen: Department of Animal Sciences-Agricultural University, 1997. (Version 2.3).
MARCONDES, C.R.; PANETO, J.C.C.; BEZERRA, L.A.F.; LÔBO, R.B. Estudo de definição alternativa da probabilidade de permanência no rebanho para a raça Nelore. Revista Brasileira de Zootecnia, v.34, p.1563-1567, 2005.

MARTINEZ, G.E.; KOCH, R.M.; CUNDIFF, L.V.; GREGORY, K.E.; VLECK, L.D. van. Genetic parameters for six measures of length of productive life and three measures of lifetime production by $6 \mathrm{yr}$ after first calving for Hereford cows. Journal of Animal Science, v.82, p.1912-1918, 2004.

MEACHAM, N.S.; NOTTER, D.R. Heritability estimates for calving date in simental cattle. Journal of Animal Science, v.64, p.701-705, 1987.

MELIS, M.H. van; ELER, J.P.; OLIVEIRA, H.N.; ROSA; G.J.M.; SILVA, J.A.V.; FERRAZ, J.B.S; PEREIRA, E. Study of stayability in Nellore cows using a threshold model. Journal of Animal Science, v.85, p.1780-1786, 2007.

PEREIRA, E.; ELER, J.P.; FERRAZ, J.B.S. Análise genética de algumas características reprodutivas e suas relações com o desempenho ponderal na raça Nelore. Arquivo Brasileiro de Medicina Veterinária e Zootecnia, v.53, p.720-727, 2001.

SAS Intitute. SAS/STAT. Cary: SAS Institute, 2000. 842p.

SILVA, J.A.I.V.; ELER, J.P.; FERRAZ, J.B.S.; GOLDEN, B.L.; OLIVEIRA, H.N. Heritability estimate for stayability in Nelore cows. Livestock Production Science, v.79, p.97-101, 2003.

SILVA, J.A.I.V.; FORMIGONI, I.B.; ELER, J.P.; FERRAZ, J.B.S. Genetic relationship among stayability, scrotal circumference and post-weaning in Nelore cattle. Livestock Science, v.99, p.51-59, 2006.

TASSELL, C.P. van; VLECK, L.D. van; GREGORY, K.E. Bayesian analysis of twinning and ovulation rates using a multiple-trait threshold model and Gibbs sampling. Journal of Animal Science, v.76, p.2048-2061, 1998.

WESTHUIZEN, R.R. van der; SCHOEMAN, S.J.; JORDAAN, G.F.; WYK, J.B. van. Heritability estimates derived from threshold analyses for reproduction and stayability traits in a beef cattle herd. South African of Animal Science, v.31, p.25-32, 2001.

Recebido em 22 de junho de 2007 e aprovado em 25 de setembro de 2007 